\title{
An Atypical Case of Pustular Psoriasis Presenting With Severe Subungual Abscesses Involving All Fingers
}

\author{
A. Tulin Mansur ${ }^{1}$ \\ 1 Dermatology Department, Baskent University Istanbul Hospital, Istanbul, Turkey \\ Key words: acrodermatitis continua, diagnosis, psoriasis, pustular psoriasis, subungual abscess \\ Citation: Mansur AT. An atypical case of pustular psoriasis presenting with severe subungual abscesses involving all fingers. Dermatol \\ Pract Concept. 2019;9(2):152-154. DOI: https://doi.org/10.5826/dpc.0902a14 \\ Accepted: October 31, 2018; Published: April 30, 2019 \\ Copyright: @2019 Mansur. This is an open-access article distributed under the terms of the Creative Commons Attribution License, which \\ permits unrestricted use, distribution, and reproduction in any medium, provided the original author and source are credited. \\ Funding: None. \\ Competing interests: The author has no conflicts of interest to disclose. \\ Authorship: The author takes responsibility for this publication. \\ Corresponding author: Prof. Dr. A. Tulin Mansur, Baskent University Istanbul Hospital, Dermatology Department, Kısıklı Cad, Oymacı \\ sok, No:7, 34662 Altunizade, Istanbul, Turkey. Email: tulinmansur@gmail.com
}

\section{Introduction}

Pustular psoriasis (PP) is a group of inflammatory skin conditions characterized by infiltration of neutrophil granulocytes in the epidermis, with clinically visible sterile pustules. Acrodermatitis continua of Hallopeau (ACH), which is now considered to be a variant of PP, is characterized by primary and persistent ( $>3$ months) pustules affecting the nail bed/matrix [1]. Here we report a patient with PP manifesting itself with acute subungual abscesses involving all fingers.

\section{Case Presentation}

A 51-year-old woman presented with rapidly developed yellow discoloration of fingernails, accompanied by severe throbbing pain. Dermatological examination revealed subungual abscesses involving all fingers, and periungual erythematous, edematous, and scaly changes extending to the proximal interphalangeal joint (Figure 1, A and B). Some fingernails showed distal onycholysis and oil spots, while distal subungual hyperkeratosis and thickening were evident on all toenails. Aerobic bacterial culture of the purulent specimen yielded Proteus spp, which was sensitive to almost all conventional antibiotics. A 1-week course of amoxicillin-clavulanate $2 \mathrm{~g} /$ day and fusidic acid ointment was unsuccessful. Proximal shedding of nails started in some of the fingers (Figure 2A), while erythematosquamous patches with superimposed grouped pustules developed on the legs (Figure 2B). Skin biopsy specimens showed parakeratosis, elongation of rete ridges, neutrophilic exocytosis, and intraepidermal pustules (Figure 3). Oral methotrexate $15 \mathrm{mg} /$ week was started. In 2 weeks, a dramatic improvement was noted (Figure 4A). At the eighth week of treatment, periungual inflammation subsided and all fingernails started to regrow (Figure 4B).

\section{Conclusions}

In $\mathrm{ACH}$, pustular eruptions tend to remain restricted to 1 or 2 digits, most commonly the thumb, for months or years, and may extend slowly to the dorsum of hands and feet. There are very limited reports of $\mathrm{ACH}$ involving more than 2 digits. Longstanding lesions may lead to anonychia and destruction of the underlying bones [1,2]. 

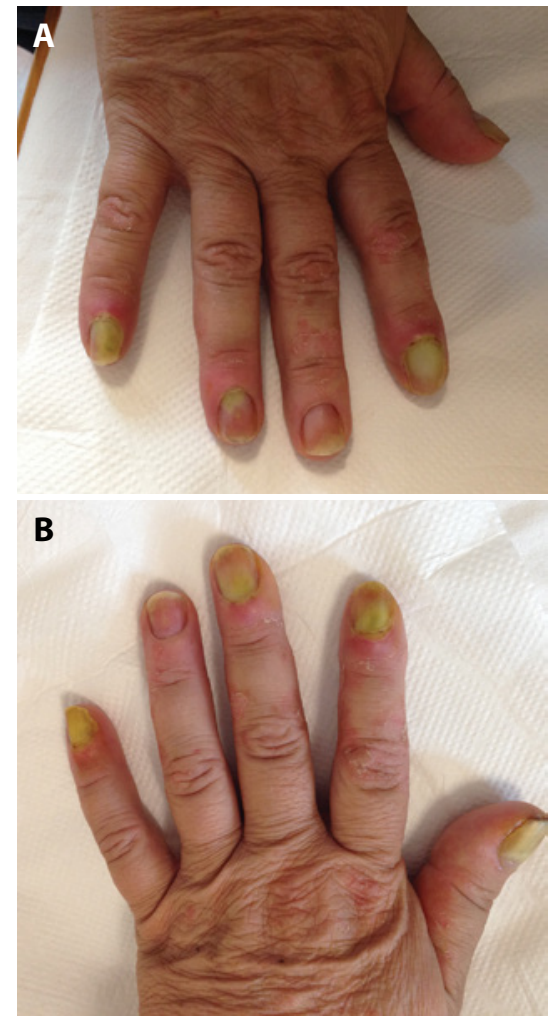

Figure 1. $(\mathrm{A}, \mathrm{B})$ Subungual abscesses on fingernails, with periungual erythema and scaling. [Copyright: (2019 Mansur.]

An acute, severe course involving almost all fingernails and causing rapid onychomadesis is unusual for $\mathrm{ACH}$, and in this context, infectious paronychia may be considered. However, bacterial abscesses developing simultaneously beneath several nails, resistant to oral and topical antibiotic therapy, should be extremely rare. Therefore, we think that in our patient the culture result merely represents a contamination or secondary infection.

Very rarely $\mathrm{ACH}$ may evolve into generalized PP in 1-2 years, mostly in elderly patients [2]. In contrast, the presented patient showed a rapid development of pustular lesions in relatively remote areas. The widespread and intense involvement of nails in addition to rapid progression and generalization does not support considering this case a typical example of $\mathrm{ACH}$.

The phenotypes of PP are not well defined, and attempts to constitute a better classification of the subgroups are ongoing [1]. The number of digits
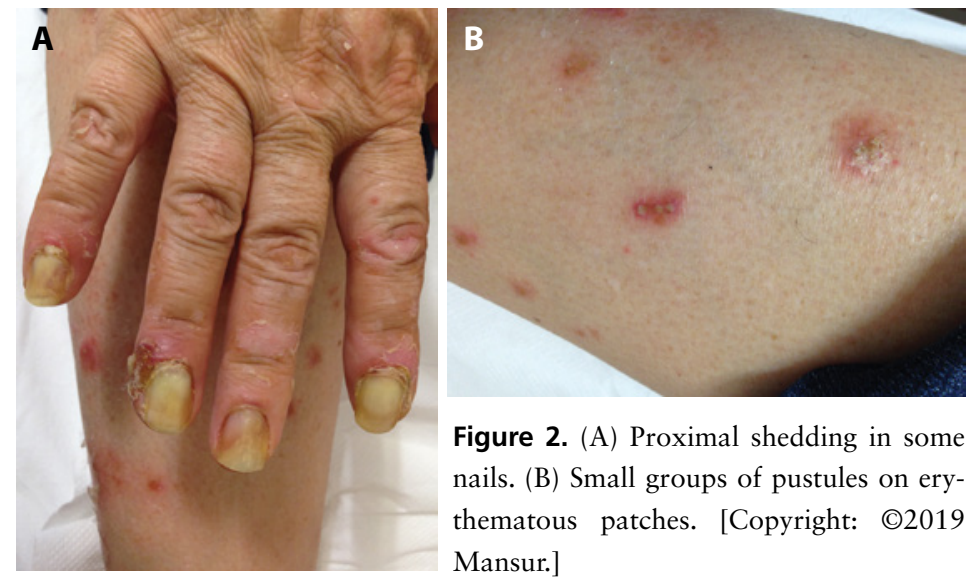

Figure 2. (A) Proximal shedding in some nails. (B) Small groups of pustules on erythematous patches. [Copyright: (O2019 Mansur.]

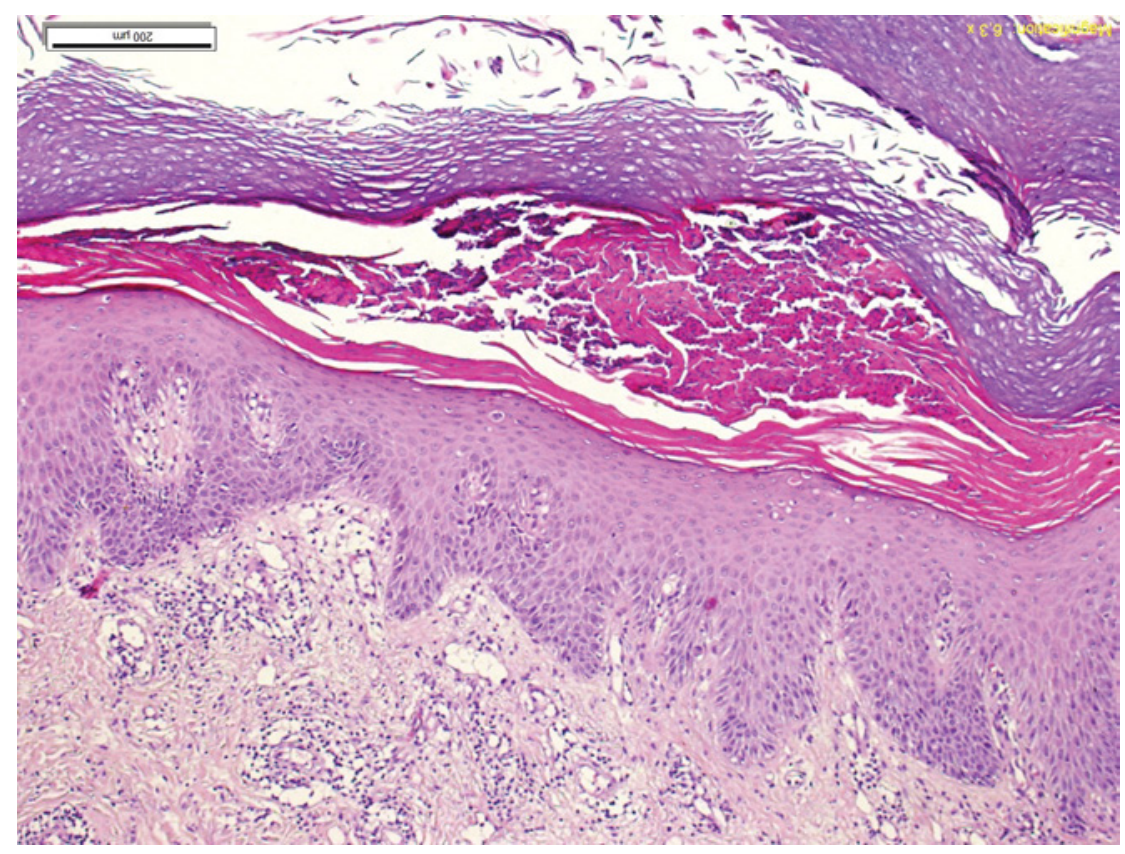

Figure 3. Histopathological features of the lesions (hematoxylin and eosin, $\times 100$ ). [Copyright: (02019 Mansur.]

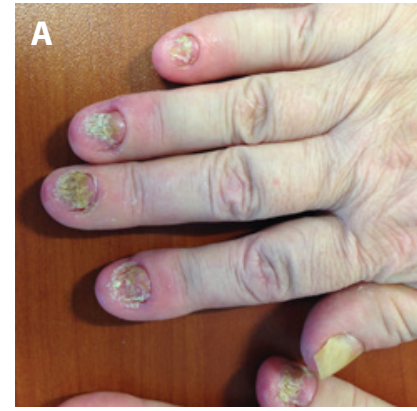

Figure 4. (A) Prominent periungual erythema and edema with total loss of fingernails. (B) Re-

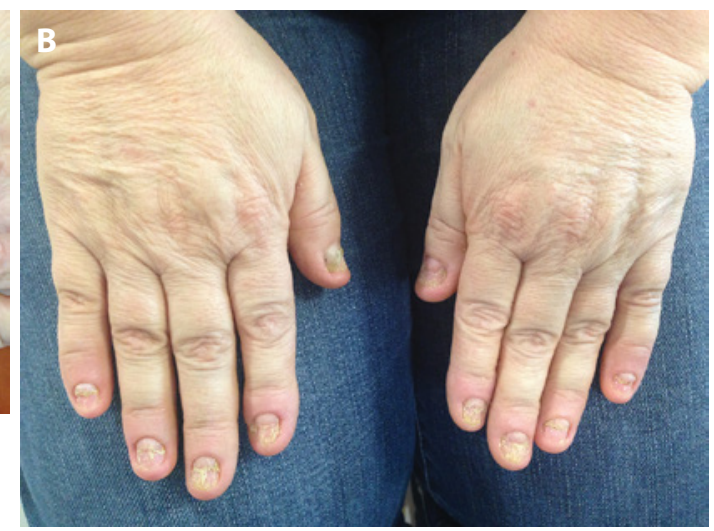

growth of nails after total shedding. [Copyright: @2019 Mansur.]

involved and the rate of progression that would ensure a precise diagnosis of ACH is not definite yet. Types of PP may not always be clearly sepa- rated from each other, and at least some patients may represent an overlapping. Accurate diagnosis and treatment is imperative for PP to avoid its detri- 
mental effects. In cases of subungual abscesses resistant to antibiotic therapy, PP involving nail apparatus should be considered.

\section{Acknowledgment}

The author thanks Tülay Zenginkinet, MD, pathologist, for helping to prepare the photomicrographs of the lesions.

\section{References}

1. Navarini AA, Burden AD, Capon F, et al. ERASPEN Network. European consensus statement on phenotypes of pustular psoriasis. J Eur Acad Dermatol Venereol. 2017;31(11):1792-1799.

2. Kim KH, Kim HL, Suh HY, et al. A case of acrodermatitis continua accompanying with osteolysis and atrophy of the distal phalanx that evoluted into generalized pustular psoriasis. Ann Dermatol. 2016;28(6):794-795. 